\title{
Automatic Recognition of the Unconscious Reactions from Physiological Signals
}

\author{
Leonid Ivonin, Huang-Ming Chang, Wei Chen, and Matthias Rauterberg \\ Eindhoven University of Technology, Eindhoven, The Netherlands \\ \{1.ivonin, h.m.chang, w. chen, g.w.m.rauterberg\}@ tue.nl
}

\begin{abstract}
While the research in affective computing has been exclusively dealing with the recognition of explicit affective and cognitive states, carefully designed psychological and neuroimaging studies indicated that a considerable part of human experiences is tied to a deeper level of a psyche and not available for conscious awareness. Nevertheless, the unconscious processes of the mind greatly influence individuals' feelings and shape their behaviors. This paper presents an approach for automatic recognition of the unconscious experiences from physiological data. In our study we focused on primary or archetypal unconscious experiences. The subjects were stimulated with the film clips corresponding to 8 archetypal experiences. Their physiological signals including cardiovascular, electrodermal, respiratory activities, and skin temperature were monitored. The statistical analysis indicated that the induced experiences could be differentiated based on the physiological activations. Finally, a prediction model, which recognized the induced states with an accuracy of $79.5 \%$, was constructed.
\end{abstract}

Keywords: Affective computing, archetypes, the collective unconscious.

\section{Introduction}

Since the beginning of the last decade affective computing has become a prominent research direction and attracted attention of researches who work on new generations of human-computer interfaces. Originally, Rosalind Picard defined affective computing as a computing that "relates to, arises from, or deliberately influences emotions" [1]. However, later affective computing gave an impulse to a more generic research area of physiological computing. The latter was introduced by Fairclough [2] and extended the scope of investigation from emotions to general psychological states of users. Physiological computing is seen as a novel mode of human-computer interaction (HCI) that enables development of computer systems, which are aware of the users' emotional and cognitive states and, thus, can dynamically adapt to their needs without the requirement of purposeful and overt communication from the users.

The research in physiological computing has built upon and confirmed many findings from psychophysiology, the field that extensively studies the physiological bases of psychological processes. In particular, it has become clear that responses of the autonomic nervous system have a good potential of being applied in computing 
applications because they are capable of predicting changes in psychological states of individuals and can be measured with relatively cheap, quick and unobtrusive methods [3]. The possible applications of physiological computing cover a range of domains and can be roughly divided into two branches: cognitive and affective. Cognitive physiological computing is directed at monitoring and improvement of the users' performance. For instance, in adaptive automation scenarios where an operator needs to control an aircraft or a vehicle, it is important to identify the states of boredom and low vigilance because they are likely to increase the risk of accidents $[4,5]$. On the other hand, affective physiological computing is aimed at increase of pleasure in interaction with computer systems and is well suited for domains such as entertainment or computer-based learning [6]. Naturally, there is an overlap between these two branches of physiological computing [3] due to the fact that cognition and affect are interrelated in the human psyche.

One course of investigation in physiological computing involves study of the psychological states that have been identified in psychology but have not yet been considered with regard to HCI. It is of little surprise that research in psychology and neuroscience has collected more knowledge about human cognition, affect and behavior than any other disciplines. For this reason, physiological computing is largely based on original experiments in psychophysiology [2] and adoption of new insights from these fields seems rational. An emerging trend in psychological science over the past 30 years is understanding and acceptance of the fact that human experience is extensively tied to a deeper level of psyche, which is not directly available to conscious awareness and, thus, defined as the unconscious. Although it may sound controversial and surprising, often people are not very well aware of and not able to accurately report on their higher order cognitive processes [7]. The absence of introspective awareness about the unconscious mental processes does not mean that they have no influence or effect on behaviors, experiences and memories. On the contrary, carefully designed experiments with both healthy volunteers and brain-damaged patients have indicated that a large part of people's everyday behaviors is conducted without any conscious control [8]. As the phenomenon of the unconscious is still to be fully understood by the scientific community, there has not been developed an established definition for it yet. However, in order to avoid ambiguity and confusion, the unconscious processes have been operationally defined by Bargh "in terms of a lack of awareness of the influences or effects of a triggering stimulus and not of the triggering stimulus itself" [9]. This definition emphasizes the important distinction between unconscious and subliminal by resolving the common confusion about these two phenomena. People outside of psychological science often equate the unconscious with processing of stimuli, which are too weak or short to enter the conscious awareness and, therefore, are referred to as subliminal. In fact, the unconscious information processing is not necessarily associated with presentations of subliminal stimuli and runs continuously as a parallel background process in human mind [10].

Carl Jung, a Swiss psychiatrist, developed his concept of the unconscious in the beginning of the previous century. According to Jung, the unconscious consists of two components: the personal unconscious and the collective unconscious. The personal unconscious is a repository for all of one's feelings, memories, knowledge and 
thoughts that are not conscious at a given moment of time [11]. They may be retrieved from the personal unconscious with a varying degree of difficulty that depends on how actively they are being repressed. On the other hand, the collective component of the unconscious is universal and has contents and modes of behavior that are uniform in all individuals [12]. The collective unconscious represents the deepest level of the psyche and does not arise individually but is inherited and contains innate behavior patterns for survival and reproduction developed over evolutionary time. The content of the collective unconscious was conceptualized by Jung as archetypes or pre-existent forms. Archetypes are very close analogies to instincts because the latter are impersonal, inherited traits that present and motivate human behavior long before any consciousness develops [13]. Furthermore, feelings and ideas emerged from archetypes continue to influence people despite any degree of consciousness later on. Archetypes define the patterns of instinctual behaviors and are conceptualized as images or representations of the instincts.

The unconscious side of the human psyche is a profoundly intriguing phenomenon guiding smart and adaptive processes that shape behaviors and experiences of people and yet remain hidden from their conscious awareness. While research in physiological computing has made a considerable progress in recognition of cognitive and affective states of the users, the investigation has been primary focused on conscious psychological states. Thus, sensing a deeper level of human experiences defined by the unconscious processes remains a largely unexplored area. Interestingly, there is some initial evidence from psychophysiology [14] that the unconscious experiences of people can be indirectly assessed with their physiological signals. This fact implies that although the unconscious processes are hidden from the conscious mind, traces of the unconscious can be observed from bodily activations. However, a further investigation is required in order to evaluate the feasibility of sensing the users' unconscious mental processes in HCI scenarios by means of physiological computing.

In this study our primary goal was the evaluation of the possibility to sense the unconscious experiences of the users in an automatic and unobtrusive manner. However, as the unconscious is a complex phenomenon, the scope of our study was limited to the collective unconscious. Unlike the personal unconscious that is highly diverse and individual, the collective unconscious consists of the universal archetypes. For this reason, it is better suited for computing applications where a range of common archetypal experiences could be employed for system adaptation to psychological states of the users. More specifically, this study was aimed at investigating the feasibility of sensing and distinguishing various archetypal experiences of the users based on the analysis of physiological signals such as heart rate or skin conductance.

In accordance with our research objectives an experiment was designed where explicit emotional feelings, such as fear or joy, and a range of common archetypal experiences were elicited in individuals. The explicit emotions were included in this study to serve as a benchmark that can be used to compare our results with other affect recognition studies. Simultaneously with the presentation of emotional and archetypal stimuli physiological signals of the participants were unobtrusively measured with a number of wireless sensors. Subsequently, signal processing methods were applied to the collected physiological data and a set of appropriate features was extracted with 
advanced data mining techniques. In order to explore the obtained dataset several statistical tests were utilized. Finally, predictive models that allow for a meaningful classification of the subjects' psychological states were constructed based on the vector of features extracted earlier.

\section{Methods}

\subsection{Experimental Design}

Stimuli. An appropriate set of stimuli was required for the elicitation of the explicit emotions and the archetypal experiences in the experiment. Past research in affect elicitation have applied different media types for emotion induction in laboratory conditions, including images and sounds [15, 16], music [17], and films [18]. These media types differ from one another in many aspects. For instance, still images and sounds are commonly presented to subjects for very short periods of time and have a high temporal resolution. On the other hand, music and film clips accommodate a lower degree of temporal resolution lasting for several minutes and deliver heterogeneous cognitive and affective activations. In comparison with the other types of media, film clips are powerful in capture of attention because of their dynamic display that includes both visual and auditory modalities [18]. Another characteristic of film clips is the ability to elicit intensive emotional responses that lead to activations in cognitive, experiential, central physiological, peripheral physiological and behavioral systems [19]. Taking into account the pros and cons of each media type film clips were chosen for this study because they effectively elicit emotions and last for several minutes. The latter fact was important for calculation of heart rate variability parameters that require at least 5 minutes of data [20]. With regard to the archetypal stimuli we assumed that the media with a high affective impact would also have a large influence on the collective unconscious. For this reason, film clips were utilized for the induction of both the explicit emotions and the archetypal experiences.

Emotions or feelings are commonly represented in affective computing with the dimensional model [21]. This model projects emotions in the affective space with two or three dimensions. In case of two dimensions an emotional state in the affective space is characterized by values of arousal and valence. The dimension of arousal ranges from calm to aroused states while the dimension of valence ranges from negative to positive states [22]. For this study five explicit emotions, amusement, fear, joy, sadness and neutral state, were selected. They uniformly cover the two-dimensional affective space. According to the previous work in this field [23], the neutral state is located close to the origin of the affective space and each one of the other four emotions is situated in a separate quadrant of the space. The film clips for elicitation of each chosen explicit emotional state were identified based on the previous studies in affect induction and recognition. The seminal work of Gross [18] and Pantic [24] provides guidance with regard to application of video in emotion research and even proposes sets of film clips that can be readily used as emotional stimuli. However, we could not always use the recommended clips for the two following reasons. First, some of the film clips were considerably shorter than 5 minutes. Second, from the 
pilot study we learnt that some of the clips taken from old movies do not emotionally engage people because they are perceived as old-fashioned. Thus, we introduced five film clips that were selected according to the requirements of this study and presented them in Table 1.

Having prepared stimuli for the explicit emotions, it was next necessary to obtain film clips for the archetypal experiences. Jung discovered that symbolic representations of archetypes had been present across cultures for thousands of years. Archetypal symbols are commonly found in artwork, myths, storytelling, and continue to be actively employed in modern mass media [25]. This fact led us to the idea that the set of stimuli could be constructed by extracting typical archetypal appearances from a variety of rich media sources. However, beforehand we had to determine which archetypes would be included in the experiment.

Table 1. Information about sources of the film clips

\begin{tabular}{cccc}
\hline Film clip & Movie & Film clip & Movie \\
\hline \multicolumn{4}{c}{ Stimuli for Explicit Emotions } \\
\hline Neutral & $\begin{array}{c}\text { Coral Sea Dreaming: } \\
\text { Awaken [26] }\end{array}$ & Fear & $\begin{array}{c}\text { The Silence of The Lambs } \\
\text { [27] }\end{array}$ \\
\hline Amusement & Mr. Bean [28] & Joy & The Lion King [29] \\
\hline Sadness & Forrest Gump [30] & \\
\hline \multicolumn{4}{c}{ Archetypal stimuli } \\
\hline Anima & American Beauty [31] & Hero Return & Braveheart [32] \\
\hline Animus & Black Swan [33] & Mentor & The King's Speech [34] \\
\hline Hero Departure & Braveheart [32] & Mother & All About My Mother [35] \\
\hline Hero Initiation & Braveheart [32] & Shadow & Fight Club [36] \\
\hline
\end{tabular}

For this study eight archetypes (anima, animus, hero-departure, hero-initiation, hero-return, mentor, mother and shadow) were selected based on their importance and representativeness. Therefore, only films depicting the most common archetypes [12] formed our set of stimuli. The archetypes of anima, animus and shadow were chosen based on their appearance in the manuscripts of Jung [13]. Three archetypes of a hero exemplify important stages in the hero's journey described by Joseph Campbell [37]. He identified that a prototypic journey, which a hero undertakes in a generic narrative, includes stages of departure, initiation, and return. The archetype of mentor is found in the research of Campbell as well and signifies a character that supports the hero in acquiring knowledge and power. Finally, mother is yet another major archetype [38] that was picked for this study.

Next, film clips embodying these eight archetypes were needed. Similar to the stimuli for the explicit emotions and to the previous studies that employed films [19] we obtained our clips by editing fragments of full-length commercial movies. However, unlike the explicit emotions, there was no guidance from the past research with regard to the selection of the stimuli. Thus, our choices had to be evaluated and, if necessary, corrected by experts in the area of archetypal research. Therefore, we pursued collaboration with The Archive for Research in Archetypal Symbolism (ARAS), which is 
an organization that since the early 1930s has been collecting and annotating mythological, ritualistic, and symbolic images from all over the world [39]. Thanks to the cooperation with ARAS and their feedback, our set of archetypal stimuli was constructed from the clips, which were obtained from the movies listed in Table 1.

Unfortunately, copies of the film clips employed in the study cannot be shared due to the fact that they were extracted from commercial movies. However, all of the movies are freely available on the market and we will provide the editing instructions to produce exactly the same clips upon request.

Participants. Thirty-six healthy volunteers were recruited for this study. Many of them were undergraduate or graduate students. Eventually, 10 participants had to be excluded from the data analysis due to technical problems with Shimmer wireless physiological sensors. One more participant was excluded because he did not comply with the procedure of the experiment and the validity of his data was questionable. For this reason, only data from 25 subjects, consisting of 12 women and 13 men, was utilized in this study. Of these, 11 participants were from Europe, 10 participants originally came from Asia, 3 participants were from Middle East and one participant was from South America. The average age for the women was 23.0 years $(\mathrm{SD}=1.9$ ) and for the men 25.4 years $(\mathrm{SD}=4.5)$. Participants had normal or corrected to normal vision and hearing. Prior to the experiment each subject signed an informed consent form and was later financially compensated for participation in the laboratory session that took approximately 2 hours.

Apparatus. The laboratory was equipped with a high definition beamer that in a cinema like settings projected the film clips on a white wall with dimensions 592 x 222 $\mathrm{cm}$. The couch that accommodated participants during the study was situated at a viewing distance of approximately 4 meters in front of the white wall. Additionally to the beamer, a computer screen and a mouse were located near the couch. After watching a film clip subjects were required to use the mouse for providing a self-report about their feelings by rating them against a number of scales, which were displayed on the screen. The procedure of the experiment including presentation of the clips, collection of the feedback, and time tracking was synchronized and automated with a website. Heart activities and skin conductance of participants were monitored with Shimmer wearable wireless sensors that streamed physiological data to a computer via Bluetooth connection. The three-lead Shimmer electrocardiogram sensor was connected with four disposable pregelled $\mathrm{Ag} / \mathrm{AgCl}$ spot electrodes. Two of the electrodes were placed below the left and right collarbones and the other two were attached to the left and right sides of the belly. The electrode placed on the right side of the belly served as a reference. The same type of electrodes was used to connect the Shimmer GSR sensor to thenar and hypothenar eminences of the participant's palm on a non-dominant hand for measurement of the skin conductance. Regrettably, due to the malfunctioning of the Shimmer ECG sensor, physiological data of 10 participants was partly missing and had to be excluded from the analysis. A Refa amplifier from TMSI BV in combination with an inductive respiration belt and a temperature 
sensor was used for the measurement of the respiration and skin temperature. A respiration belt with an appropriate size was strapped around the participant's chest and the temperature sensor was fixed on the subject's belly with a sticky tape.

Procedure. Each participant went through a session of the experiment individually. Upon arrival to the laboratory, a participant was invited to sit upright on the couch. Then, the participant was asked to read and sign the provided informed consent form. Next, the experimenter demonstrated the required positioning of the physiological sensors on a body, assisted the participant to attach them, and ensured that the sensors streamed signals of good quality. After placement of the sensors the experimenter allowed a time interval of approximately five minutes to pass before presentation of the first film clip. This interval was necessary for the electrode gel to soak into the participant's skin and thereby establish a stable electrical connection [40]. Meanwhile, an overview of the study was given to the subject. The overview further clarified the procedure of the study explaining that several film clips would be played, and the participant's physiological signals would be continuously recorded during the film's demonstration. However, the actual goal of the experiment remained undisclosed and, for this reason, the participant was not aware of any emotions or archetypes pictured in the clips. Following the overview, the subject was asked to make her comfortable on the couch and refrain from unnecessary movements during the session. The light in the laboratory was dimmed so that the viewing experience became similar to the one in a movie theater. The demonstration of the film clips always started with the neutral film (Coral Sea Dreaming: Awaken [26]) because the participants had to be brought to the same psychophysiological baselines. Piferi et al. [41] argued that a relaxing aquatic video could be used for establishing the baseline. Then, the other film clips were shown in random order. A short video demonstrating a breathing pattern preceded presentation of each film clip (including the neutral one). This video lasted for 40 seconds and its purpose was to dismiss psychological and physiological effects of the previous stimuli. During this video the participant was required to follow the breathing pattern and thereby adjust her respiration rate to the common baseline. Upon completion of viewing a film clip, the participant provided a retrospective self-report by rating her feelings along a number of dimensions using the computer screen and the mouse located near the participant's dominant hand. The discussion of the data collected in self-reports is out of the scope of this manuscript and will be presented elsewhere. As soon as the participant submitted the self-report for the last film clip, the light in the room was turned on and the experimenter helped the subject with detaching the physiological sensors from her body. Finally, the participant was debriefed and reimbursed.

Physiological Signals. A number of physiological signals were monitored in this study. The decision regarding inclusion of a particular signal in the experiment was made based on the background literature in psychophysiology and will be further discussed in this section. 
Electrocardiogram (ECG) is a measurement of the heart's electrical activity conducted with electrodes attached to the skin surface and recorded over a period of time. ECG was monitored at $512 \mathrm{~Hz}$ and then cleaned with low-pass, high-pass, and notch filters. ECG contains plenty of information about the cardiovascular activity, and in the psychophysiological domain it is commonly used for the calculation of the heart rate (HR) and heart rate variability (HRV). The heart rate is a simple measurement that characterizes the heart's activity in terms of the number of heart beats per minute [42]. The HR was obtained from the ECG signal by identifying beats with an algorithm provided in [43] and computing the average heart rate over a moving window of 10 seconds. We expected to see a relation between the psychological states of the subjects and their HR because this measure had been widely applied in physiological computing and, according to Kreibig [44], the HR is the most often reported cardiovascular measure in psychophysiological studies of emotion. Next, several HRV parameters from time and frequency domains were calculated based on the heart beats data with an HRVAS software package [45]. Time domain parameters included the standard deviation of the beat to beat intervals (SDNN), the square root of the mean of the sum of the squares of differences between adjacent beat to beat intervals (RMSSD), and the standard deviation of differences between adjacent beat to beat intervals (SDSD) [20]. A pool of frequency domain parameters consisted of a total power, a power in a very low frequency range (VLF, 0-0.04 Hz), a power in a low frequency range $(\mathrm{LF}, 0.04-0.15 \mathrm{~Hz})$, a power in a high frequency range $(\mathrm{HF}, 0.15-0.4$ $\mathrm{Hz}$ ), and a ratio of the power in a low frequency range to the power in a high frequency range (LF/HF) [20].

Skin conductance describes variations in the electrodermal activity of skin and is associated with processes of eccrine sweating, which are controlled by the sympathetic branch of the autonomic nervous system [40]. According to [23], skin conductance is closely related to psychological processes and particularly to the level of arousal. Skin conductance has tonic and phasic components. The tonic component reflects relatively slow changes in skin conductance over longer periods of time lasting from tens of seconds to tens of minutes. Thus, it is indicative of a general level of arousal and is known as the skin conductance level (SCL). A different perspective is given by the phasic component of skin conductance, which is called the skin conductance response (SCR), because it reflects high frequency variations of the conductance and is directly associated with observable stimuli [40]. The skin conductance signal was recorded at $512 \mathrm{~Hz}$. Although such a high sampling rate is not imperative for measurement of the skin conductance signal, complex analysis approaches and smoothing procedures can benefit from higher resolution data [40]. The SCL was obtained from the raw skin conductance signal by applying a low pass filter at $1 \mathrm{~Hz}$. An additional high pass filter was set at $0.5 \mathrm{~Hz}$ for the SCR.

Respiration is yet another physiological signal that has been often studied in psychophysiology [46]. This signal is correlated with processes in the sympathetic nervous system and is indicative of psychological states of individuals [47]. The raw respiration signal was monitored at $512 \mathrm{~Hz}$ and treated with low pass and high pass filters. Then, the respiration rate (RR) was obtained from the signal based on the 
guidelines provided by the manufacturer of the respiration sensor (TMSI BV). Afterwards, the RR was averaged with a moving window of 10 seconds.

Skin temperature (ST) fluctuates due to localized variations in the blood flow characterized by vascular resistance or arterial blood pressure that are in turn modulated by the sympathetic nervous system [48]. It has been previously reported in literature [49] that affective stimuli can cause variations in ST of individuals. The ST signal was monitored at $512 \mathrm{~Hz}$. However, the raw data was later harmlessly resampled to $64 \mathrm{~Hz}$ because it is a slow changing signal. High frequency noise was eliminated with a low pass filter of $10 \mathrm{~Hz}$ that was applied to the resampled signal. Finally, the signal was smoothed with a moving window of 10 seconds.

Keeping in mind practical HCI applications, we preferred not to include electroencephalography (EEG) measures in this study due to concerns about the robustness of the EEG signal.

\subsection{Statistical Analysis}

As stated in the introduction, one of the motivations for this study was the question whether the patterns of physiological responses to various archetypal experiences are different and, furthermore, if the difference is statistically significant. We were also interested how physiological activations modulated by the explicit emotions of the participants are different comparing to their responses elicited by the archetypal stimuli. A number of statistical tests had to be conducted in order to answer these questions.

Each subject watched all the film clips that formed our sets of stimuli for the explicit emotions and the archetypal experiences. Thus, the study had repeated-measures design where physiological measurements were made on the same individual under changing experimental conditions. An appropriate statistical test for this type of design would be multivariate analysis of variance (MANOVA) for repeated measures [50]. However, certain assumptions of this test were violated for some of the physiological signals' features in our study. Namely MANOVA does not allow inclusion of time-varying covariates in the model and an unequal number of repeated observations per an experimental condition. The former requirement could not be fulfilled because the physiological baselines that were introduced to the statistical model as covariates consisted of multiple data points. Although this assumption could easily be satisfied by transforming a number of data points into a single feature, we preferred to preserve the richness of our dataset and refrained from, for instance, averaging the baseline record. The latter prerequisite of MANOVA demands an equal number of repeated measurements per experimental condition. It could not be met due to the fact that the film clips presented during the experiment had slightly different length and, consequently, the size of vectors with physiological data varied. While all the clips lasted for approximately 5 minutes, there was a considerable difference between some of the stimuli. The shortest film clip had duration of 4 minutes and 46 seconds whereas the longest one was 6 minutes and 35 seconds.

The limitations of MANOVA can be overcome if the statistical analysis is performed with linear mixed models (LMM). LMMs are parametrical statistical models 
for clustered, longitudinal or repeated-measures data that characterize the relationships between continues dependent variables and predictor factors [51]. LLMs have another advantage over MANOVA - they allow participants with missing data points to be included in the analysis. In contrast, MANOVA drops the entire dataset of a subject even if just one data point is absent. The general specification of an LMM for a given participant $i$ can be defined as follows:

$$
Y_{i}=X_{i} \beta+Z_{i} u_{i}+\varepsilon_{i}
$$

In this equation $Y_{i}$ is a vector of continues responses for the $i$-th subject and $X_{i}$ is a design matrix that contains values of the covariates associated with the vector of fixed-effect parameters $\beta$. The $Z_{i}$ matrix is comprised of covariates that are associated with random effects for the $i$-th subject. The vector or random effects is assumed to follow a multivariate normal distribution and is denoted with $u_{i}$. Finally, the $\varepsilon_{i}$ vector represents residuals. A more elaborate introduction into LMMs can be found in, for instance, [51] or [52].

A software implementation of statistical procedures included in SPSS Version 19 (SPSS, Inc.) was utilized to answer the research questions pointed out earlier. Physiological responses of the subjects were treated as dependent variables (continuous responses), the film clips represented fixed variables and the physiological baselines measured during the presentation of the video with a breathing pattern before each stimulus were used as covariates. The LMMs main effect tests whether the patterns of the participants' physiological responses are different between various stimuli. The HRV features were analyzed with MANOVA as they met the requirements of this method. All statistical tests used a 0.05 significance level.

\subsection{Data Mining Techniques}

The statistical analysis can enable us to determine whether or not it is possible to distinguish the archetypal experiences of people based on the patterns of their physiological activations corresponding to each of the archetypes. However, a statistically significant difference between the physiological responses associated with various archetypes does not allow evaluation of the practical feasibility to accurately predict psychological states of the participants. On the other hand, a prediction model that maps physiological signals and the unconscious states of the users is what will be appreciated by HCI practitioners. Thus, besides the statistical analysis, data mining techniques were applied to the dataset in order to obtain a predictive model that would facilitate evaluation of the classification accuracy among the archetypal experiences. In order to make physiological data from different individuals comparable, the baseline values were subtracted from the data corresponding to stimuli presentations. The result of the subtraction was then normalized to a range from 0 to 1 for each subject separately.

Classification Methods. As demonstrated film clips had a duration of approximately 5 minutes, the physiological data that was recorded for each stimulus formed temporal 
sequences. The time sequences classification is different from the recognition of static information due to the increased complexity of the classification task. In general three main kinds of sequence classification algorithms can be distinguished [53] as shown below. However, there are also alternative approaches (see, for instance, [54]).

- Feature based classification essentially transforms a sequence classification problem to a representation amenable for conventional classification methods such as decision trees and neural networks.

- Sequence distance based classification relies on a distance measure that defines the similarity between a pair of time sequences. Similar to the feature based classification the idea of this method is to translate a sequence classification task into a domain where some existing classification algorithm can be used.

- Model based classification makes an assumption that time series are generated by an underlying model. This method requires a statistical model that given a class of data sequences defines the probability distribution of the sequences in the class [53].

In physiological computing, the feature based method of time sequence classification currently dominates [3]. From our point of view, this approach was also well tailored to this study. It has several advantages comparing to the other classification methods. First, this method provides a convenient way to include non-temporal attributes, such as some of the HRV features or the gender of subjects, into the analysis, which sequence distance or model approaches do not [55]. Second, contrary to HMM, this method does not require a considerable amount of training data in order to demonstrate a satisfying performance [55]. Taking into consideration the fact that the physiological data of 10 participants had to be discarded from the analysis, the quantity of data was clearly not enough to achieve competitive classification accuracy with HMM. Third, the identification of model streams representing typical time series that correspond to various psychological states is not trivial in the sequence distance method.

After the selection of attributes, several classifiers were used to construct predictive models for classification. In our analysis 5 classification methods frequently used in physiological computing [3] were evaluated. K-nearest neighbor (kNN) is a simple algorithm that performs instance-based learning classifying an object based on the classes of its neighbors. The second classifier was support vector machine (SVM) that constructs a set of hyperplanes for classification purposes. The third classification method relied on a probabilistic model built with the naïve Bayes algorithm. The fourth approach was linear discriminant analysis (LDA) that is well suited for small data samples and is easy in implementation [3]. Finally, the fifth classification method was the $\mathrm{C} 4.5$ algorithm for generation of decision trees. The decision trees were used in conjunction with Adaptive Boosting (AdaBoost) [56] in order to achieve higher accuracy. It was important to guarantee that the classification algorithms are not trained and tested on the same dataset because we wanted to obtain subject independent results. Therefore a leave-one-out cross-validation technique was employed for assessments of the classification performance. 
Selection of Features. An essential prerequisite of the classification is the extraction of feature vectors from data sequences. The main goal pursued by the extraction of features is a compression of data sequences to smaller sets of static features. The sliding window, the Discrete Wavelet Transform (DWT) and the Discrete Fourier Transform (DFT) [57-59] are three methods for conversion of time series to static data. The sliding window method performs best with low frequency and relatively short time sequences because an increase of the signal's frequency and length leads to generation of high dimensional feature vector. For long and high frequency data series the DWT and DFT approaches have been introduced. The idea behind these methods is the transformation of a sequence from the time domain to the time-frequency plane (DWT) or to the frequency domain respectively (DFT). Taking into consideration the aspects of our setup, the sliding window method for extraction of feature vectors was an appropriate way to prepare the dataset for the classification. Another name of this approach is segmentation since it first involves partition of a time axis into multiple segments with equal length and then averaging of temporal data along the segments [59].

Our next step was to use the segmentation method with the collected physiological data. The ECG signal provided 38 features in total: 30 features were obtained from the HR temporal data by averaging values of the HR along the segments of 10 seconds; 8 features of the HRV signal (SDNN, RMSSD, SDSD, total power, VLF, $\mathrm{LF}, \mathrm{HF}$, and $\mathrm{LF} / \mathrm{HF}$ ). A total of 60 features were extracted from the skin conductance signal. The first 30 features belonged to the SCL signal that was averaged over 30 segments of 10 seconds each. The remaining 30 features were generated in a similar manner from the SCR signal. Then, the respiration signal was converted to 30 features representing the average RR calculated on each of the segments. Another 30 features were obtained from the ST signal by calculating average values over the 30 segments. Finally, we had 158 features ready for the classification.

Dimension Reduction. Reduction of dimensionality is a recommended step in the data mining process. There are various techniques for the reduction of features including principal component analysis (PCA) and LDA. For the purposes of our study LDA was chosen over PCA because in general PCA has the weakness of only capitalizing on between-class information, while LDA uses both within- and between-class information for better performance [60]. Two aspects of LDA should be mentioned here. First, strictly speaking, LDA is not a feature selection but a feature extraction method that obtains the new attributes by a linear combination of the original dimensions. The reduction of dimensionality is achieved by keeping the components with highest variance. Second, LDA can be used for both the identification of important features and classification [3]. Dimension reduction with LDA reduced 158 features into 7 components. For illustration of the importance of each physiological signal in the extracted components, coefficients of determination $\left(R^{2}\right)$ were calculated and put in Table 2. The coefficients of determination specify the amount of variance in one of the 7 discriminant functions that can be described by all the features of a certain 
physiological signal. The data in Table 2 suggests that electrocardiography and skin conductivity were two measurements which contributions to the discriminant functions were the strongest.

Table 2. Coefficients of determination $\left(R^{2}\right)$ for the seven discriminant functions

\begin{tabular}{ccccccccc}
\hline & 1 & 2 & 3 & 4 & 5 & 6 & 7 & Average \\
\hline ECG & 0.553 & 0.228 & 0.607 & 0.971 & 0.742 & 0.905 & 0.184 & 0.599 \\
\hline SC & 0.098 & 0.060 & 0.151 & 0.098 & 0.164 & 0.299 & 0.507 & 0.197 \\
\hline RR & 0.003 & 0.000 & 0.003 & 0.005 & 0.006 & 0.001 & 0.000 & 0.003 \\
\hline ST & 0.004 & 0.030 & 0.004 & 0.000 & 0.006 & 0.056 & 0.002 & 0.015 \\
\hline
\end{tabular}

\section{Results}

\subsection{Statistical Analysis}

The initial motivation of this study was to explore the relationships between the archetypal experiences and their physiological correlations. The statistical analysis was to answer the question whether the archetypal experiences of the participants elicited with the film clips have a significant effect on their physiological signals. The features extracted from ECG, skin conductance, respiration and skin temperature recordings were arranged to form three types of datasets: one with the data for the explicit emotions, another with the data for the archetypal experiences and the unified dataset.

LMMs were fit to each of the datasets with the HR features. The analysis, which the HR entered as a dependent variable, demonstrated a significant interaction effect between the film clips and the HR baselines for all the datasets: the explicit emotions dataset, $[\mathrm{F}(4,541.443)=2.513, \mathrm{p}=0.041]$, the archetypal experiences dataset $[\mathrm{F}(7$, $1028.618)=3.503, \mathrm{p}=0.001]$ and the unified dataset, $[\mathrm{F}(12,1521.573)=3.929, \mathrm{p}<=$ $0.001]$.

As the HRV features were calculated over the whole duration of every stimulus and were represented with a single data point, they could be easily analyzed with MANOVA for repeated measures. This test showed a significant main effect of the film clips on the HRV parameters of the participants' physiological responses for two of the datasets: the explicit emotions dataset, $[\mathrm{F}(32,329.811)=2548, \mathrm{p}<=0.001$ (Wilks' lambda)] and the unified dataset, $[\mathrm{F}(96,1903.193)=1987, \mathrm{p}<=0.001$ (Wilks' lambda)]. However, the same test for the archetypal experiences dataset was not significant, $[\mathrm{F}(56,872.323)=1281, \mathrm{p}=0.085$ (Wilks' lambda) $]$.

The relationship between the SCL features and the presentations of the stimuli was investigated with LMMs. The statistical tests indicated a significant interaction effect between the film clips and the SCL baselines for the explicit emotions dataset $[\mathrm{F}(4$, $2884,487)=42.130, \mathrm{p}<=0.001]$, the archetypal experiences dataset $[\mathrm{F}(7,5880.869)$ $=38.795, \mathrm{p}<=0.001]$ and the unified dataset $[\mathrm{F}(12,9868.854)=27.615, \mathrm{p}<=0.001]$. Next, we ran analysis for the SCR features in a similar manner. A significant interaction effect between the film clips and the baseline covariates was discovered for the 
explicit emotions dataset, $[\mathrm{F}(4,707.582)=13.473, \mathrm{p}<=0.001]$, the archetypal experiences dataset, $[\mathrm{F}(7,1391.923)=11.401, \mathrm{p}<=0.001]$ and the unified dataset, $[\mathrm{F}(12$, 2109.957) $=10.667, \mathrm{p}<=0.001]$.

Then, we looked at the respiration data and performed tests with LMMs that were fit to the RR measurements. The interaction between the film clips and the baseline RR did not demonstrated significance for the archetypal experiences dataset, $[\mathrm{F}(7$, $1071.446)=1.070, \mathrm{p}=0.380]$ and the unified dataset $[\mathrm{F}(12,1686.540)=1.667, \mathrm{p}=$ $0.068]$. Nevertheless, the same test was significant for the explicit emotions dataset, $[\mathrm{F}(4,611.304)=2.931, \mathrm{p}=0.020]$.
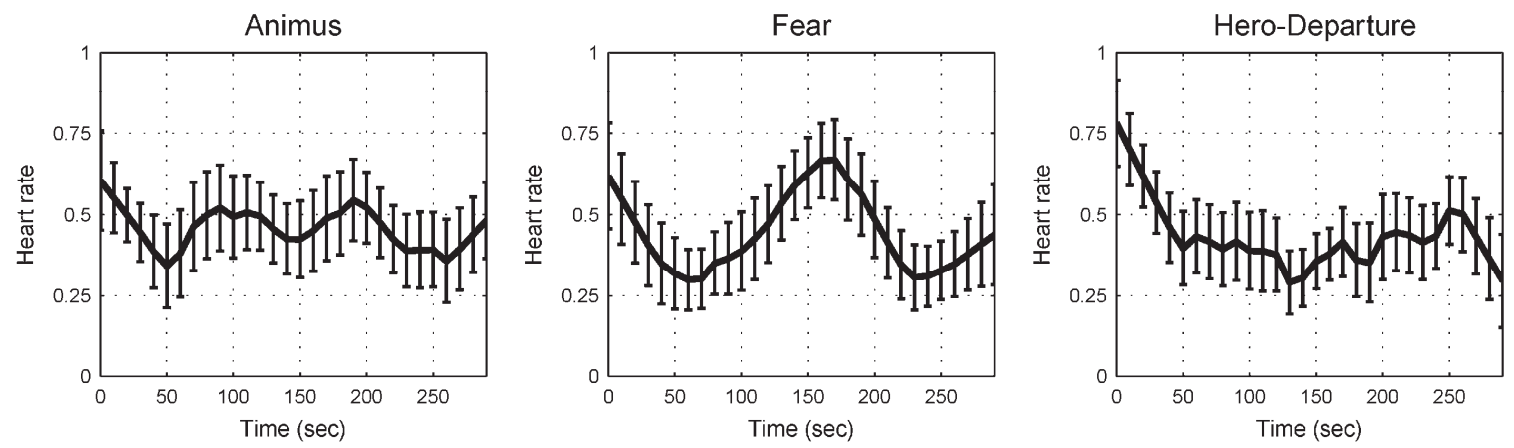

Fig. 1. Heart rate responses of the subjects to the film clips. The mean values and 95\% confidence intervals of the HR are represented with the bold lines and the vertical bars.

Finally, the features of the skin temperature recordings were analyzed. Again, LMMs built on the ST data were used for the statistical testing. However, we could not complete the analysis because the statistical software did not achieve a convergence within 100 of iterations.

For illustrative purposes, the data of HR signal that contributed to the discriminant functions the most is presented on Fig. 1. The mean values and 95\% confidence intervals of the HR are indicated for several of the stimuli.

\subsection{Classification}

After the statistical analysis an evaluation of several predictive models was conducted. This evaluation was aimed at answering the question of how accurate the archetypal experiences can be predicted and classified by computational intelligence algorithms from physiological data.

Similar to the statistical analysis the classification was performed on three collections of data records: the explicit emotions dataset, the archetypal dataset and the unified dataset that integrated all the available data. Every selected classification method (kNN, SVM, naïve Bayes, LDA and AdaBoost with decision trees) was applied to each of the datasets.

For the archetypal dataset the model constructed with the $\mathrm{kNN}$ method was able to correctly classify $74 \%$ of the instances. However, the same classification approach resulted in the recognition rate of $72 \%$ for the explicit emotions dataset and of $49.8 \%$ for the unified dataset. The number of nearest neighbors equal to $20(k=20)$ lead to 
the optimal performance in all the cases. The SVM algorithm provided better classification accuracy than $\mathrm{kNN}$ for the archetypal dataset $(75.5 \%)$ and for the unified dataset $(68.3 \%)$. On the other hand it demonstrated slightly lower recognition rate on the explicit emotions dataset (71.2\%). The naïve Bayes and LDA approaches enabled us to achieve similar performance on the archetypal dataset $(79.5 \%)$ and the explicit emotions dataset $(74.4 \%)$. The recognition rate on the unified dataset was higher with the LDA method (61.2\%) comparing to the naïve Bayes classifier (57.2\%). Finally, decision trees in conjunction with AdaBoost led to the poorest classification results: $67 \%$ for the archetypal dataset, $68.8 \%$ for the explicit emotions dataset and $47.1 \%$ for the unified dataset. A summary of the classification results is provided in Table 3.

Table 3. Classification performance achieved with different methods for the archetypal, the explicit and the unified datasets

\begin{tabular}{cccccc}
\hline Dataset & kNN & SVM & Naïve Bayes & LDA & AdaBoost \\
\hline Archetypal & $74 \%$ & $75.5 \%$ & $79.5 \%$ & $79.5 \%$ & $67 \%$ \\
\hline Explicit & $72 \%$ & $71.2 \%$ & $74.4 \%$ & $74.4 \%$ & $68.8 \%$ \\
\hline Unified & $49.8 \%$ & $68.3 \%$ & $57.2 \%$ & $61.2 \%$ & $47.1 \%$ \\
\hline
\end{tabular}

\section{Discussion}

\subsection{Statistical Analysis}

A number of statistical tests were run on the collected data. Their outcomes gave evidence of a significant relationship between some of the physiological signals and the psychological conditions of the subjects. Whereas the patterns in three out of the four measurements reflected the induced explicit emotions, no association could be inferred from the skin temperature signal. These findings were anticipated and go along with the state of the art of physiological computing. Unfortunately, the skin temperature signal did not justify our expectations and, from our point of view, its variations are too slow to successfully contribute to the differentiation of emotions. The archetypal states of the participants demonstrated statistically significant relationship with the HR, SCL and SCR features extracted from the ECG and skin conductance signals. In comparison with the explicit emotions, the archetypal experiences lead to observable activations in a smaller number of the physiological features. Nevertheless, our results show that the patterns of physiological responses to various archetypal experiences are different and the difference is statistically significant. Furthermore, the analysis performed with the unified dataset, which integrated the explicit emotions and the archetypal experiences, supported the hypothesis of possibility to distinguish between these two types of stimuli.

\subsection{Classification Accuracy}

Having conducted the statistical analysis we were curious how accurately computational intelligence methods can classify the archetypal experiences. The findings of 
our study indicate that prediction models built with data mining techniques and trained on the physiological data of the subjects achieved reasonably high precision. Five different classification methods were used to obtain the models that demonstrated classification rates from $67 \%$ to $79.5 \%$. These results are not subject-depended and characterize the ability of the models to predict the correct archetypal conditions from the physiological recordings of an unknown user. While it is easy to check the classification accuracy of the explicit emotions against other studies in affect recognition, the same comparison for the archetypal experiences is challenging. We are aware of only one study [61] where the responses of people to brief presentations of mandala symbols, which are considered to express the archetype of the self, were measured with physiological sensors. The reported classification rate of $23.3 \%$ is considerably lower than the results obtained in this study. This fact can be explained with the differences in the design of the experiments. In particular, the duration of stimuli presentation and the type of stimuli seem to be important. Indeed, the film clips extracted from blockbuster movies are likely to be more powerful than the images of mandala presented for several seconds. Our findings for the archetypal experiences can also be set against previous studies in affect recognition just to have a relative benchmark. Judging by the review provided in [3], the classification accuracy of our models is on par with the ones demonstrated in those studies. Although in some cases higher recognition rates have been reported, for instance in [62-64] researchers achieved classification precision of up to $97.4 \%$, two types of limitations, which seem to exist in these studies, have to be taken into account. First, in some cases the classification is subject-dependent, meaning that recognition algorithms are adjusted to perform well only with the data belonging to a particular individual and cannot be generalized. Second, the number of the psychological conditions that are being predicted is important because the more classes to be classified, the more difficult the problem becomes. This can be illustrated with a simple example - in a case of two classes problem, accuracy of $50 \%$ is attained simply by chance, while in a situation with 8 classes the chance level drops to $12.5 \%$. In our experiment we had 8 conditions to be differentiated, but the best recognition rates were obtained in the studies that considered only 2 or 3 affective states.

Looking at the obtained results it seems clear that the Naïve Bayes and LDA classification methods generally outperformed other approaches. A particularly interesting finding is that higher prediction accuracy was obtained for the archetypal dataset comparing to the explicit emotions dataset. The better recognition rate was achieved despite of the fact that the archetypal dataset contained 8 classes while the explicit emotion dataset only 5. From our point of view, the archetypes were classified more accurately than the explicit emotions because by definition they elicit cognitive and affective activations that are universal across the population. On the other hand, the explicit emotions are more subject-dependent and considerably vary due to an individual's personality.

In the future work it will be necessary to improve the classification accuracy. From our point of view, an increase of the recognition rate can be achieved by taking into account additional parameters of physiological signals and performing subjectdependent classification. 


\subsection{Archetypal Stimuli}

The stimuli for induction of the archetypal experiences were a crucial component of the study. However, as it was the first experiment where the archetypal experiences were elicited with film clips, we did not have an empirical evidence that the stimuli we identified with the help of ARAS [39] would successfully express the 8 targeted archetypes. Another concern was related to the length of the film clips. Due to the fact that they each lasted approximately five minutes, it was reasonable to expect highly heterogeneous affective and cognitive responses of the participants that would complicate the recognition problem. Judging by our findings, the selection of the film clips was satisfactory and the universal unconscious reactions in subjects from various regions of the world were elicited successfully. This conclusion can be made based on the accurate recognition rate that otherwise would not be possible to attain.

\subsection{Limitations}

Several limitations of this study should be highlighted. First, a relatively small number of participants can be considered as a limitation. Next, during the presentation of the film clips the subjects generally sit still and the amount of movements was small. For this reason, we did not have to implement any dedicated signal processing methods for combating the movement artifacts that would likely be necessary in many HCI scenarios. The third limitation is the generalizability of the results. Only a single example of each archetype was presented to the participants. This fact limits the generalizability of our findings and demands new studies with more representations for every archetype to be carried out. Finally, it is necessary to perform comparison of the physiological data and the self-reports provided by the subjects. Such a comparison will indicate to which extent the participants were able to consciously perceive the archetypal appearances in the film clips.

\subsection{Conclusion}

To a large extent people are driven by the collective unconscious in their decisions, motivations, and behaviors. So far, the unconscious experiences of the users have received little attention in physiological computing because it has been primary dealing with cognition and affect. In this study the feasibility of recognizing the archetypal experiences of users, which constitute the collective unconscious, with wireless sensors and without human interventions, was evaluated. The experiment that featured 8 archetypes visualized with the film clips was executed in order to collect physiological data. We then applied data mining methods to the obtained dataset, performed statistical analysis and built several prediction models that demonstrated prediction accuracy of up to $79.5 \%$. Thus, physiological sensors coupled with computational intelligence algorithms can facilitate development of HCI interfaces that sense archetypal experiences of the users and use this information for system adaptation.

Acknowledgements. The authors thank The Archive for Research in Archetypal Symbolism (ARAS) and in particular John Beebe, Baruch Gould, Iden Goodman, Allison Tuzo and Tom Singer for the help with identification and selection of the archetypal 
stimuli. We also thank the anonymous reviewers for their helpful comments. This work was supported in part by the Erasmus Mundus Joint Doctorate (EMJD) in Interactive and Cognitive Environments (ICE), which is funded by Erasmus Mundus under the FPA no. 2010-2012.

\section{References}

1. Picard, R.W.: Affective computing, MIT Media Laboratory Perceptual Computing Section Technical Report No. 321 (1995)

2. Fairclough, S.H.: Fundamentals of physiological computing. Interacting with Computers 21, 133-145 (2009)

3. Novak, D., Mihelj, M., Munih, M.: A survey of methods for data fusion and system adaptation using autonomic nervous system responses in physiological computing. Interacting with Computers 24, 154-172 (2012)

4. Zhou, F., Qu, X., Helander, M.G., Jiao, J.(R.): Affect prediction from physiological measures via visual stimuli. International Journal of Human-Computer Studies 69, 801-819 (2011)

5. Wu, D., Courtney, C.G., Lance, B.J., Narayanan, S.S., Dawson, M.E., Oie, K.S., Parsons, T.D.: Optimal arousal identification and classification for affective computing using physiological signals: virtual reality stroop task. IEEE Transactions on Affective Computing 1, 109-118 (2010)

6. Stickel, C., Ebner, M., Steinbach-Nordmann, S., Searle, G., Holzinger, A.: Emotion detection: application of the valence arousal space for rapid biological usability testing to enhance universal access. In: Stephanidis, C. (ed.) Universal Access in HCI, Part I, HCII 2009. LNCS, vol. 5614, pp. 615-624. Springer, Heidelberg (2009)

7. Nisbett, R.E., Wilson, T.D.: Telling more than we can know: verbal reports on mental processes. Psychological Review 84, 231-259 (1977)

8. Van Gaal, S., Lamme, V.A.F.: Unconscious high-level information processing: implication for neurobiological theories of consciousness. The Neuroscientist 18, 287-301 (2012)

9. Bargh, J.A., Morsella, E.: The unconscious mind. Perspectives on Psychological Science 3, 73-79 (2008)

10. Rauterberg, M.: Emotions: The voice of the unconscious. In: Yang, H.S., Malaka, R., Hoshino, J., Han, J.H. (eds.) ICEC 2010. LNCS, vol. 6243, pp. 205-215. Springer, Heidelberg (2010)

11. Sally, W.: Algorithms and archetypes: evolutionary psychology and Carl Jung's theory of the collective unconscious. Journal of Social and Evolutionary Systems 17, 287-306 (1994)

12. Jung, C.G.: The archetypes and the collective unconscious. Princeton University Press, Princeton (1981)

13. Jung, C.G.: Man and his symbols. Doubleday, Garden City (1964)

14. Miller, N.E.: Some examples of psychophysiology and the unconscious. Applied Psychophysiology and Biofeedback 17, 3-16 (1992)

15. Lang, P.J., Bradley, M.M., Cuthbert, B.N.: International affective picture system (IAPS): affective ratings of pictures and instruction manual. Technical Report A-8, Gainesville, FL, USA (2008)

16. Bradley, M.M., Lang, P.J.: International affective digitized sounds (IADS): stimuli, instruction manual and affective ratings (Tech. Rep. No. B-2), Gainesville, FL, USA (1999)

17. Eich, E., Ng, J.T.W., Macaulay, D., Percy, A.D., Grebneva, I.: Combining music with thought to change mood. In: Coan, J.A., Allen, J.J.B. (eds.) The Handbook of Emotion Elicitation and Assessment, pp. 124-136. Oxford University Press, New York (2007) 
18. Gross, J.J., Levenson, R.W.: Emotion elicitation using films. Cognition \& Emotion 9, 87-108 (1995)

19. Rottenberg, J., Ray, R.D., Gross, J.J.: Emotion elicitation using films. In: Coan, J.A., Allen, J.J.B. (eds.) Handbook of Emotion Elicitation and Assessment, pp. 9-28. Oxford University Press, New York (2007)

20. Task Force of the European Society of Cardiology and the North American Society of Pacing and Electrophysiology: Heart rate variability: standards of measurement, physiological interpretation, and clinical use. Circulation 93, 1043-1065 (1996)

21. Russell, J.A.: A circumplex model of affect. Journal of Personality and Social Psychology 39, 1161-1178 (1980)

22. Ivonin, L., Chang, H.-M., Chen, W., Rauterberg, M.: A new representation of emotion in affective computing. In: Proceeding of 2012 International Conference on Affective Computing and Intelligent Interaction (ICACII 2012), Taipei, Taiwan. Lecture Notes in Information Technology, pp. 337-343 (2012)

23. Lang, P.J., Greenwald, M.K., Bradley, M.M., Hamm, A.O.: Looking at pictures: affective, facial, visceral, and behavioral reactions. Psychophysiology 30, 261-273 (1993)

24. Soleymani, M., Pantic, M., Pun, T.: Multimodal emotion recognition in response to videos. IEEE Transactions on Affective Computing 3, 211-223 (2011)

25. Faber, M.A., Mayer, J.D.: Resonance to archetypes in media: there is some accounting for taste. Journal of Research in Personality 43, 307-322 (2009)

26. Hannan, D.: Coral sea dreaming: awaken. Roadshow Entertainment (2010)

27. Demme, J.: The silence of the lambs. Orion Pictures (1991)

28. Atkinson, R., Curtis, R.: Mr. Bean (season 1, episode 1). Pearson Television International (1990)

29. Allers, R., Minkoff, R.: The Lion King. Walt Disney Pictures (1994)

30. Zemeckis, R.: Forrest Gump. Paramount Pictures (1994)

31. Mendes, S.: American beauty. DreamWorks Pictures (1999)

32. Gibson, M.: Braveheart. 20th Century Fox (1995)

33. Aronofsky, D.: Black swan. Fox Searchlight Pictures (2010)

34. Hooper, T.: The king's speech. The Weinstein Company (2010)

35. Almodóvar, P.: All about my mother. Warner Sogefilms (1999)

36. Fincher, D.: Fight club. 20th Century Fox (1999)

37. Campbell, J.: The hero with a thousand faces. New World Library, Novato (2008)

38. Maloney, A.: Preference ratings of images representing archetypal themes: an empirical study of the concept of archetypes. Journal of Analytical Psychology 44, 101-116 (2002)

39. Gronning, T., Sohl, P., Singer, T.: ARAS: archetypal symbolism and images. Visual Resources 23, 245-267 (2007)

40. Figner, B., Murphy, R.O.: Using skin conductance in judgment and decision making research. In: Schulte-Mecklenbeck, M., Kuehberger, A., Ranyard, R. (eds.) A Handbook of Process Tracking Methods for Decision Research, pp. 163-184. Psychology Press, New York (2011)

41. Piferi, R.L., Kline, K.A., Younger, J., Lawler, K.A.: An alternative approach for achieving cardiovascular baseline: viewing an aquatic video. International Journal of Psychophysiology 37, 207-217 (2000)

42. Neuman, M.R.: Vital signs: heart rate. IEEE Pulse 1, 51-55 (2010)

43. Afonso, V.X., Tompkins, W.J., Nguyen, T.Q.: ECG beat detection using filter banks. IEEE Transactions on Biomedical Engineering 46, 192-202 (1999)

44. Kreibig, S.D.: Autonomic nervous system activity in emotion: a review. Biological Psychology 84, 394-421 (2010) 
45. Ramshur, J.T.: Design, evaluation, and application of heart rate variability software (HRVAS). Master's thesis, The University of Memphis, Memphis, TN (2010)

46. Fairclough, S.H., Venables, L.: Prediction of subjective states from psychophysiology: a multivariate approach. Biological Psychology 71, 100-110 (2006)

47. Boiten, F.A.: The effects of emotional behaviour on components of the respiratory cycle. Biological Psychology 49, 29-51 (1998)

48. Kim, K.H., Bang, S.W., Kim, S.R.: Emotion recognition system using short-term monitoring of physiological signals. Medical \& Biological Engineering \& Computing 42, 419-427 (2004)

49. Ekman, P., Levenson, R., Friesen, W.: Autonomic nervous system activity distinguishes among emotions. Science 221, 1208-1210 (1983)

50. O'Brien, R.G., Kaiser, M.K.: MANOVA method for analyzing repeated measures designs: an extensive primer. Psychological Bulletin 97, 316-333 (1985)

51. West, B.T., Welch, K.B., Galecki, A.T.: Linear mixed models: a practical guide using statistical software. Chapman and Hall/CRC, Boca Raton (2006)

52. Cnaan, A., Laird, N.M., Slasor, P.: Using the general linear mixed model to analyse unbalanced repeated measures and longitudinal data. Statistics in Medicine 16, 2349-2380 (1997)

53. Xing, Z., Pei, J., Keogh, E.: A brief survey on sequence classification. ACM SIGKDD Explorations Newsletter 12, 40 (2010)

54. Holzinger, A., Stocker, C., Bruschi, M., Auinger, A., Silva, H., Gamboa, H., Fred, A.: On applying approximate entropy to ECG signals for knowledge discovery on the example of big sensor data. In: Huang, R., Ghorbani, A.A., Pasi, G., Yamaguchi, T., Yen, N.Y., Jin, B. (eds.) AMT 2012. LNCS, vol. 7669, pp. 646-657. Springer, Heidelberg (2012)

55. Kadous, M.W., Sammut, C.: Classification of multivariate time series and structured data using constructive induction. Machine Learning 58, 179-216 (2005)

56. Freund, Y., Schapire, R.E.: A decision-theoretic generalization of on-line learning and an application to boosting. Journal of Computer and System Sciences 55, 119-139 (1997)

57. Agrawal, R., Faloutsos, C., Swami, A.: Efficient similarity search in sequence databases. In: Lomet, D.B. (ed.) FODO 1993. LNCS, vol. 730, pp. 69-84. Springer, Heidelberg (1993)

58. Chan, F.K.: Haar wavelets for efficient similarity search of time-series: with and without time warping. IEEE Transactions on Knowledge and Data Engineering 15, 686-705 (2003)

59. Geurts, P.: Pattern extraction for time series classification. In: Siebes, A., De Raedt, L. (eds.) PKDD 2001. LNCS (LNAI), vol. 2168, pp. 115-127. Springer, Heidelberg (2001)

60. Martinez, A.M., Kak, A.C.: PCA versus LDA. IEEE Transactions on Pattern Analysis and Machine Intelligence 23, 228-233 (2001)

61. Ivonin, L., Chang, H.-M., Chen, W., Rauterberg, M.: Unconscious emotions: quantifying and logging something we are not aware of. Personal and Ubiquitous Computing 17, 663-673 (2013)

62. Healey, J.A., Picard, R.W.: Detecting stress during real-world driving tasks using physiological sensors. IEEE Transactions on Intelligent Transportation Systems 6, 156-166 (2005)

63. Picard, R.W., Vyzas, E., Healey, J.: Toward machine emotional intelligence: analysis of affective physiological state. IEEE Transactions on Pattern Analysis and Machine Intelligence 23, 1175-1191 (2001)

64. Sakr, G.E., Elhajj, I.H., Huijer, H.A.-S.: Support vector machines to define and detect agitation transition. IEEE Transactions on Affective Computing 1, 98-108 (2010) 